\title{
An Estimation-Range Extended Autocorrelation-Based Frequency Estimator
}

\author{
Cui Yang, Gang Wei, and Fang-jiong Chen \\ School of Electronic and Information Engineering, South China University of Technology, 381 Wushan Road, \\ Guangzhou 510640, China \\ Correspondence should be addressed to Cui Yang, yangcui26@163.com
}

Received 24 June 2009; Revised 26 August 2009; Accepted 19 October 2009

Recommended by Erchin Serpedin

\begin{abstract}
We address the problem of autocorrelation-based single-tone frequency estimation. It has been shown that the frequency can be estimated from the phase of the available signal's autocorrelation with fixed lag. A large lag results in better performance but at the same time limits the estimation range. New methods have been proposed to extend the estimation range. In this paper, a new estimator which is a robust hybrid of periodogram-based and autocorrelation-based frequency estimators is presented. We propose to calculate the autocorrelation function with spectral lines inside the available signal's main lobe spectrum. We show that the new estimator obtains full estimation range of $[-\pi, \pi)$. The theoretical performance bound is also deduced. Performance analysis and simulations demonstrate that the proposed estimator approaches the CRLB.
\end{abstract}

Copyright ( 12009 Cui Yang et al. This is an open access article distributed under the Creative Commons Attribution License, which permits unrestricted use, distribution, and reproduction in any medium, provided the original work is properly cited.

\section{Introduction}

The problem of estimating the frequency of a complex exponential from a finite number of samples in additive white noise arises in many fields including radar, sonar, measurement, wireless communications, and speech processing [1-16]. For instance, frequency estimation of singletone sinusoidal signals is an important technique for carrier recovery in wireless communication systems $[6,10]$.

Many techniques have been proposed for frequency estimation over the years. Rife [1] proposed the optimal Maximum Likelihood (ML) estimator, which is to locate the peak of a periodogram. The estimator achieves asymptotic unbiased estimation and its mean square error (MSE) approaches the CRLB when the signal-to-noise ratio (SNR) is larger than a certain value. However, the ML estimator is not computationally simple [14]. Suboptimal algorithms with lower computation have been proposed, such as the linear prediction-based estimators $[2,3]$, the autocorrelation-based estimators [4-11], and the periodogram-based estimators [12-14]. The linear prediction algorithms are to estimate the frequency from the coefficients of the predictor. The autocorrelation algorithms are to extract the frequency from the phase of the available signal's autocorrelation with fixed lags. The periodogram-based estimators use the Discrete Fourier Transform (DFT) for a coarse search and an interpolation technique for a fine search.

We focus on the autocorrelation-based algorithms in this paper. The autocorrelation of a noiseless single-tone complex sinusoidal signal can be presented as $R(\tau)=A^{2} \exp (j \omega \tau)$, where the phase contains the unknown frequency. Various techniques have been proposed to estimate the frequency from the phase component. But these techniques perform quite differently in MSE, complexity, and estimation range. The estimator solely based on $R(1)$ [3] achieves full estimation range of $-\pi \leq \omega<\pi$ but its MSE performance is not satisfactory [16]. The estimator based on $\tau>1$ [4] can improve the MSE performance [16], but at the same time it limits the estimation range to $-\pi / \tau \leq \omega<\pi / \tau$. Fitz [5] and Luise and Reggiannini [6] proposed to average over multiple lags, which significantly improve the MSE performance. However, its estimation range is still limited by the applied maximal lag. The estimator proposed in [7] has similar MSE performance and estimation range as Fitz and Luise and Reggiannini's estimators but has lower computational complexity. Estimators in [8-11] are proposed to achieve wider acquisition range. In this paper, we approximate the original signal with the spectral lines inside the main lobe 
of the zero-padded measurements' DFT spectrum and then calculate the autocorrelation function based on the approximated signal. Since the spectral lines around the actual tone are used, only the noise inside the narrow band is important for the estimator's performance. A closed-form estimator solely based on the DFT coefficients is then derived. The proposed estimator is a robust hybrid of periodogram-based and autocorrelation-based estimators. Theoretical analysis shows that its MSE performance is independent of the correlation lags. Therefore, we can choose $\tau=1$ to obtain the full estimation range. Theoretical analysis also shows that the upper bound of its MSE is 1.3 times of the CRLB.

\section{Problem Statement}

The set of given samples $x(n)$ is modeled as

$$
x(n)=s(n)+z(n)=A e^{j(\omega n+\varphi)}+z(n),
$$

where $s(n)$ is an exponential signal, $A, \omega$, and $\phi$ are, respectively, amplitude, frequency, and original phase. $z(n)$ is zero-mean white Guassian noise with variance of $\sigma_{n}^{2}$. With the definition of autocorrelation function $\widehat{R}(\tau)=1 /(N-$ $\tau) \sum_{n=\tau}^{N-1} x(n) x^{*}(n-\tau)$, we can obtain $R(\tau)$ in noiseless cases:

$$
R(\tau)=A^{2} e^{j \omega \tau}
$$

It can be observed that the phase of $R(\tau)$ contains the unknown frequency. Thus, frequency can be resolved with the $\tau$ th correlation lag:

$$
\widehat{\omega}=\frac{1}{\tau} \arg \hat{R}(\tau)
$$

The estimators proposed by Fitz [5] and Luise and Reggiannini [6] are the weighted average of (3). For those estimators, if a small $\tau$ is used, a great performance gap can be observed when they are compared to the CRLB. An increased value of $\tau$ leads to a reduced error variance, where the reduction in error variance is especially pronounced for low SNR scenarios [16]; but meanwhile limits their estimation range $[5,6]$. Applying the main lobe spectrum approximation to the autocorrelation based algorithms, we deduce our estimator which avoids the above problems.

\section{Improved Autocorrelation-Based Frequency Estimator}

Rather than calculating autocorrelation function directly with the signal samples, we estimate it in frequency domain. Let $N$ and $M$ denote the number of available samples and the number of zero-padded samples, respectively. Assume that $X_{k}$ stands for the DFT transform of $x(n)$ and the spectral line with the highest magnitude locates at $k_{0}$. Since the power of the sinusoidal signal is mainly inside the main lobe $\left[k_{0}-\Delta, k_{0}+\Delta\right]$ while the power of white noise distributes uniformly in the whole spectrum, we adopt the idea of the main lobe approximation [15] to obtain the estimated autocorrelation function:

$$
\widehat{R}_{m \_k_{0}}(\tau)=\sum_{k=k_{0}-\Delta}^{k_{0}+\Delta}\left|X_{k}\right|^{2} e^{j k \omega_{0} \tau},
$$

where $\omega_{0}=2 \pi / M$. Substituting (4) into (3), we have the estimator

$$
\widehat{\omega}=\frac{1}{\tau} \arg \left(\sum_{k=k_{0}-\Delta}^{k_{0}+\Delta}\left|X_{k}\right|^{2} e^{j k \omega_{0} \tau}\right),
$$

where $\widehat{\omega} \in\left[\left(2 \pi k_{0} / M\right)-\left(\omega_{0} / 2\right),\left(2 \pi k_{0} / M\right)+\left(\omega_{0} / 2\right)\right)$ and $|\widehat{\omega}|<$ $\pi / \tau$. To reduce the effect of $\tau$ on the estimation range, we divide the exponential part in (5) into two parts to achieve

$$
\widehat{\omega}=\frac{1}{\tau} \arg \left(\sum_{k=-\Delta}^{\Delta}\left|X_{k+k_{0}}\right|^{2} e^{j\left(k+k_{0}\right) \omega_{0} \tau}\right) .
$$

Define $\omega_{\mathrm{c}}=\omega-(2 \pi / M) k_{0}$. Hence, with (6), the estimator based on Main Lobe Autocorrelation Function (MLAF) is achieved in a two-step process:

$$
\begin{gathered}
\widehat{\omega}=\frac{2 \pi}{M} k_{0}+\widehat{\omega}_{c} \\
\widehat{\omega}_{c}=\frac{1}{\tau} \arg \left(\sum_{k=-\Delta}^{\Delta}\left|X_{k+k_{0}}\right|^{2} e^{j k \omega_{0} \tau}\right) \triangleq \frac{1}{\tau} \arg \left(\hat{R}_{m_{-} 0}(\tau)\right)
\end{gathered}
$$

where $\hat{R}_{m_{-} 0}(\tau)$ is actually the estimated autocorrelation lag of the single tone whose frequency is $\omega_{c}$. The first step is a coarse estimation to search $k_{0}$ in the DFT spectrum and the second step described by (8) is a fine estimator based on the spectrum lines inside the main lobe. It can be observed that the coarse estimation is independent of $\tau$ and solely dependent on the location of the maximal spectral line. The effect of $\tau$ on fine estimation will be discussed in the next section.

The idea of narrow band autocorrelation estimation can also be applied to the estimators proposed in $[5,6]$.

\section{Performance Analysis and Discussion}

Given the assumption that the right $k_{0}$ is chosen, the error mainly results from the fine estimation process, including error caused by noise and main lobe autocorrelation estimation. Below we evaluate the performance of the fine estimation process with both the expectation and the mean square error.

The $\tau$ th correlation lag without narrow band approximation can be given by

$$
R_{m_{-} 0}(\tau)=\sum_{k \in[0, M-1]}\left(a_{k}^{2}+b_{k}^{2}\right) e^{j\left(k-k_{0}\right) \omega_{0} \tau},
$$

where $a_{k}$ and $b_{k}$ are the real and imaginary parts of the DFT coefficients of $s(n)$, respectively. According to (2), we have

$$
e^{j \omega_{c} \tau} R_{m_{-} 0}(0)=R_{m_{-} 0}(\tau) .
$$


Let $\delta_{a_{k}}$ and $\delta_{b_{k}}$ stand for the real and imaginary parts of the DFT coefficients of the noise, respectively. The estimated autocorrelation function is

$$
\widehat{R}_{m_{-} 0}(\tau)=\sum_{k=-\Delta}^{\Delta}\left(\left(a_{k+k_{0}}+\delta_{a_{k+k_{0}}}\right)^{2}+\left(b_{k+k_{0}}+\delta_{b_{k+k_{0}}}\right)^{2}\right) e^{j k \omega_{0} \tau} .
$$

Unwrapping (11) and substituting (9) and (10) into it, we have

$$
\begin{gathered}
\hat{R}_{m_{\_} 0}(\tau)=R_{m_{-} 0}(0) e^{j\left(\omega_{c}+\beta\right) \tau}, \\
\beta=\frac{1}{\tau} \arg \left(1+P_{\tau}+Q_{\tau}\right),
\end{gathered}
$$

where

$$
\begin{aligned}
P_{\tau} & =\frac{\sum_{k=-\Delta}^{\Delta}(\mathfrak{A}) e^{j\left(k \omega_{0}-\omega_{c}\right) \tau}}{\sum_{k=0}^{M-1}\left(a_{k}^{2}+b_{k}^{2}\right)}, \\
Q_{\tau} & =\frac{-\sum_{k=0, k \notin\left[k_{0}-\Delta, k_{0}+\Delta\right]}^{M-1}\left(a_{k}^{2}+b_{k}^{2}\right) e^{j\left(\left(k-k_{0}\right) \omega_{0}-\omega_{c}\right) \tau}}{\sum_{k=0}^{M-1}\left(a_{k}^{2}+b_{k}^{2}\right)},
\end{aligned}
$$

where $\mathfrak{A}$ denotes $2 a_{k+k_{0}} \delta_{a_{k+k_{0}}}+2 b_{k+k_{0}} \delta_{b_{k+k_{0}}}+\delta_{a_{k+k_{0}}}^{2}+\delta_{b_{k+k_{0}}}^{2}$. Substituting (12) into (8), we can achieve that the estimation error equals to $\beta$. Next, we make some approximations to simplify the argument operation in (13). Since the power of sinusoidal signal mainly distributes inside the main lobe, for rectangular window we have $\Delta=M / N$. Furthermore, the power of the white noise inside the narrow band is quite small compared with the power of the signal inside the main lobe. Hence, we have $\left(P_{\tau}+Q_{\tau}\right) \ll 1$. Replacing $\left(P_{\tau}+Q_{\tau}\right)$ by its Taylor series truncated to linear term, we have $\beta \cong$ $(1 / \tau)\left(P_{\tau, i}+Q_{\tau, i}\right)$ according to the appendix of [9], where $P_{\tau, i}$ and $Q_{\tau, i}$ are, respectively, the imaginary parts of $P_{\tau}$ and $Q_{\tau}$. The expectation of the estimation error is given by

$$
E\left[\widehat{\omega}_{c}-\omega_{c}\right] \cong \frac{1}{\tau} E\left(P_{\tau, i}+Q_{\tau, i}\right) .
$$

It can be observed from (14), (15), and (16) that the estimation error contains two parts. One is $P_{\tau, i}$, which is generated by noise and the other is $Q_{\tau, i}$, which is caused by main lobe autocorrelation estimation. Only under the condition that there is no noise and $\omega$ is a multiple of $\omega_{0}$, the estimation is unbiased. Otherwise, estimation error always exists. Thus the mean square error is given by

$$
E\left[\left(\hat{\omega}_{c}-\omega_{c}\right)^{2}\right] \cong \frac{1}{\tau^{2}} E\left[P_{\tau, i}^{2}\right]+\frac{1}{\tau^{2}} Q_{\tau, i}^{2}
$$

Define signal-to-noise ratio (SNR) as $\gamma=A^{2} / \sigma_{n}^{2}$. Given the assumption of $M=q N$, and carrying out some necessary manipulations (see Appendices A and B), we achieve

$$
\begin{gathered}
\frac{1}{\tau^{2}} E\left[P_{\tau, i}^{2}\right]<2\left(\frac{8 q+4}{\gamma N^{3} q^{2}}+\frac{8}{\gamma}\left(\frac{((q-1 / 2) \mathfrak{a}) / \pi \lambda}{N^{3} q}\right)\right) \\
\frac{1}{\tau^{2}} Q_{\tau, i}^{2} \approx \frac{4}{q^{2} N^{2}}\left(\sum_{k=-q}^{q} \frac{q \sin ^{2}(\pi(k-\alpha) / q)}{(k-\alpha)}\right)^{2},
\end{gathered}
$$



FIgURE 1: MSE of the MLAF-based estimator with different $q, N=$ $256, \tau=1$. Signal frequency randomly distributes between $[0, \pi)$.

where $\mathfrak{a}$ denotes $\sum_{\lambda=1}^{2 q} \sin (2 \pi \lambda / q) \cos (\pi \lambda / q)$ and $\alpha$ is defined in Appedix B.

Observing (17), (18), and (19), $1 / \tau^{2} E\left[P_{\tau, i}^{2}\right]$ is a function of SNR while $1 / \tau^{2} Q_{\tau, i}^{2}$ is independent of SNR. It can be calculated from (18) that when $q \geq 4$, the upper bound of $1 / \tau^{2} E\left[P_{\tau, i}^{2}\right]$ keeps constant at about 1.3 times of the CRLB. Meanwhile, a large $q$ is helpful to reduce $1 / \tau^{2} Q_{\tau, i}^{2}$. If $N$ is in the order of $10^{2}$ (e.g., 128) and $q \geq 4,1 / \tau^{2} Q_{\tau, i}^{2}$ is in the order of $10^{-8}$, which can be further reduced by increasing $q$. For low to medium SNR, such error can be ignored compared with $1 / \tau^{2} E\left[P_{\tau, i}^{2}\right]$. So we suggest choosing the parameters as $q \geq 4$ and $N$ in the order of $10^{2}$.

According to (7), (8), (18), and (19), the performance of the MLAF-based estimator is independent of $\tau$. Thus we suggest using $\tau=1$ to obtain the full estimation range of $\hat{\omega} \in[-\pi, \pi)$.

\section{Simulations}

5.1. Effect of $q$ and $\tau$. We first evaluate the effect of $q$ for the proposed estimator. Both the theoretical results and computer simulations are given in Figure 1. It can be seen that when $q \geq 4$ (e.g., $q=4,6$ ) the performance approaches the CRLB. When $q=2$, the performance gap increases with the increase of SNR. If a large $q$ is used, the error caused by noise is more significant than the error by main lobe estimation. So in practice, we suggest choosing $q \geq 4$. For rather low SNRs, the performance deviates significantly from the CRLB because of the wrong choice of $k_{0}$.

Next, we discuss the effect of $\tau$. As shown in Figure 2, we compare the MLAF-based estimator with Lank's estimator [4]. Although the performance of Lank's estimator is improved with the increase of $\tau$, the performance of 
TABLE 1: Number of complex-valued multiplications/additions and phase calculations for estimators.

\begin{tabular}{|c|c|c|c|c|c|}
\hline Estimator & DFT & Complex-valued Multiplications & Complex-valued Additions & Phase Calculations & Estimation Range \\
\hline MLAF-based & $M$ point & $2 q+1$ & $2 q$ & 1 & {$[-\pi, \pi)$} \\
\hline$[9]$ & - & $(N-1) \log _{2} N-3$ & $2 N-2 \log _{2} N-2$ & $\log _{2} N-1$ & {$[-\pi, \pi)$} \\
\hline WNALP[11] & - & $(2 N-N / 2+9) N / 4$ & $(2 N-N / 2+3) N / 4$ & 1 & {$[-\pi, \pi)$} \\
\hline Y. C. Xiao [7] & - & 6 & $4 m+6$ & 3 & {$[-\pi / m, \pi / m)$} \\
\hline WAE-subopt [8] & - & $N K(3 K+1) / 2(2 K+1)$ & $N K(3 K+1) / 2(2 K+1)$ & $K$ & {$[-\pi, \pi)$} \\
\hline$[12]$ & $N$ point & 2 & - & - & {$[-\pi, \pi)$} \\
\hline$[13]$ & $N$ point & $2(Q+1) N$ & $(Q+1)(N-1)$ & - & {$[-\pi, \pi)$} \\
\hline [14] & $N$ point & $Q(4 N+1)$ & $2 Q N$ & - & {$[-\pi, \pi)$} \\
\hline
\end{tabular}

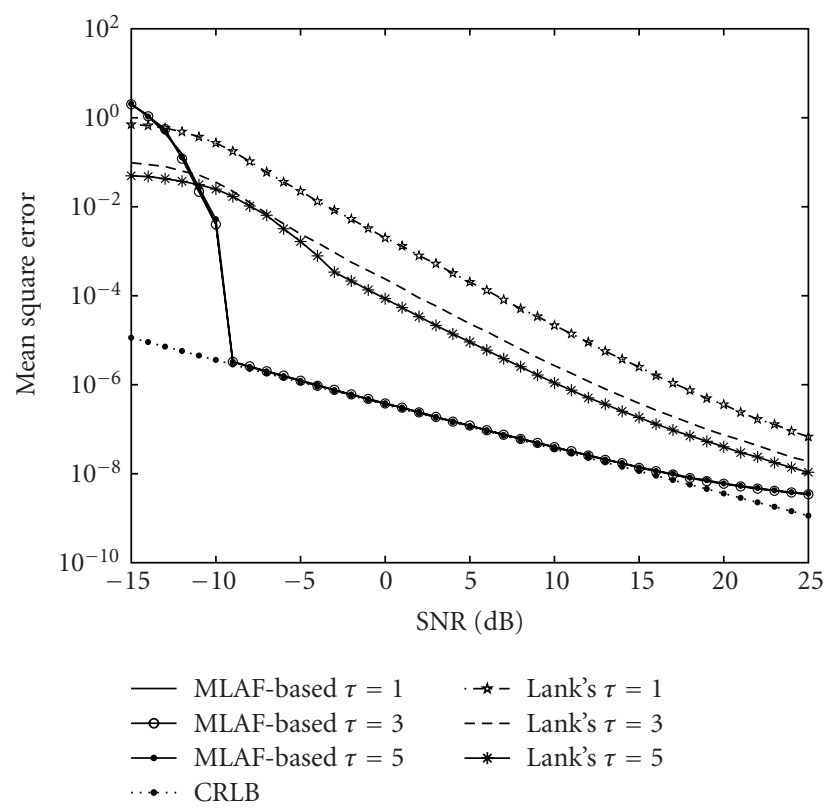

FIGURE 2: MSE of the MLAF-based estimator and Lank's estimator with different $\tau, N=256, q=4$. Signal frequency randomly distributes between $[0, \pi / 12]$.

our MLAF-based estimator keeps good and is independent of $\tau$. It also can be observed that the performance of Lank's estimator is improved when the SNR increases, while for the proposed estimator, the performance of it keeps constant for high SNRs. It is because that for high SNRs, the estimation error of the proposed estimator is mainly caused by the narrowband approximation. Such estimation error is independent of the SNR. But it can be reduced if a larger $q$ is chosen.

5.2. Comparison with Other Autocorrelation-Based Estimators. We compare the MLAF-based estimator with the iterative estimator proposed by Brown and Wang [9], the WNALP [11], and the WAE-subopt in [8] in two cases. The sample size $N$ is set to $N=24$ and $N=256$, respectively. The results are shown in Figures 3 and 4. For each case, 10000 independent runs are averaged. The estimator proposed in [7] is also simulated in Figure 4. Parameters for WAE-subopt

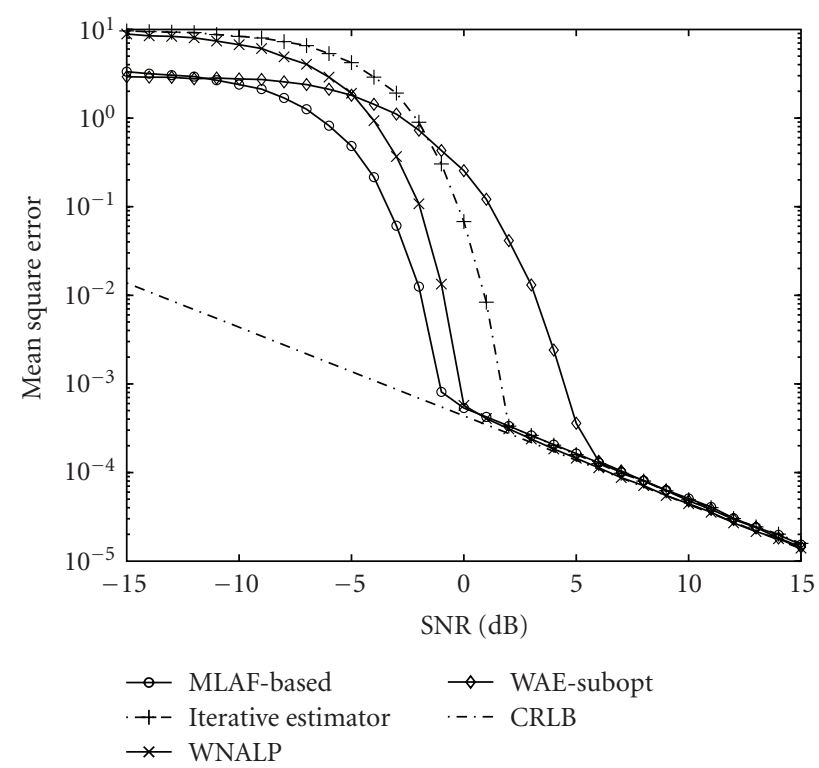

FIgURE 3: Comparison with other autocorrelation-based estimators. $N=24, \omega=0.8 \pi$. For the MLAF-based estimator, $q=4$ and $\tau=1$.

are set as $K=2, L=\{5,9\}$ in the first case and $L=\{51,103\}$ in the second case (where $K$ is the number of correlation lags and $L$ is the set of correlation lags [8]).

Comparing Figures 3 and 4, we can see that although the iterative estimator, the WNALP, and the WAE-subopt have full estimation range as the MLAF-based estimator, they have higher SNR thresholds in both the cases. We also verified that for the Y.C.X. estimator in [7], once the frequency is out of its acquisition frequency range, it can no longer operate. The phenomenon also exists for estimators proposed in $[5,6]$.

5.3. Comparison with Periodogram-Based Estimators. The proposed estimator is compared with the estimator proposed by Quinn in [12] and two estimators proposed by Aboutanios $[13,14]$. All these estimators use DFT as a coarse frequency estimation. The numerical computations for these estimators are summarized in Table 1. We can see in Figures 5 and 6 that the MLAF-based estimator has a lower SNR threshold than estimators in $[12,14]$. The estimator in [13] 


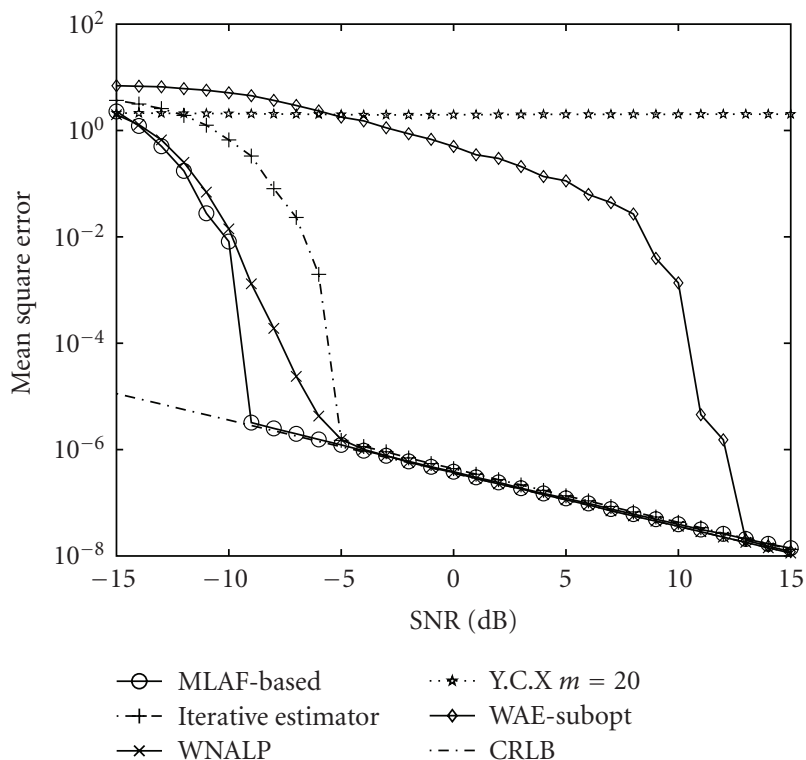

Figure 4: Comparison with other autocorrelation-based estimators. Signal frequency randomly distributes between $[0,0.8 \pi] . N=$ 256. For the MLAF-based estimator, $q=4$ and $\tau=1$.

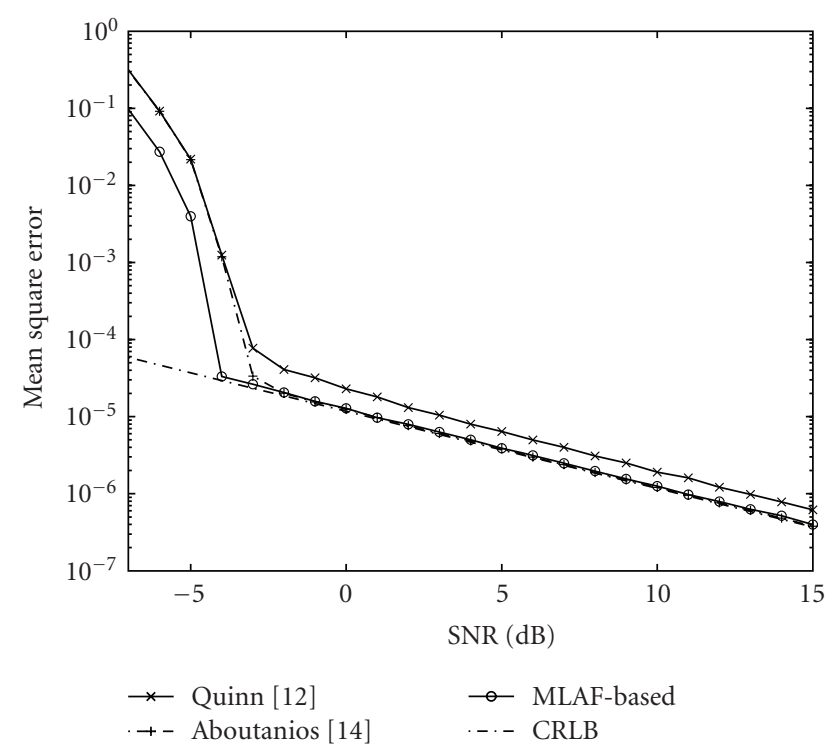

FIGURE 5: Comparison with periodogram-based estimators. Signal frequency randomly distributes between $[0,0.5 \pi] . N=80$. For the MLAF-based estimator, $q=4$ and $\tau=1$.

performs better if more iterations are applied (e.g., $Q=$ 10 , where $Q$ stands for iterations), but more iterations will induce more computations. If an $M$-point DFT is used in the coarse estimation for the estimator in [13], its SNR threshold will be the same as the proposed one. But in this case its overall complexity could be much larger because of its large complexity in the fine estimation (see Table 1).

Although the proposed estimator has to perform $M$ ponit $(M \geq 4 N)$ Fourier Transform to achieve the desired performance while the others may perform $N$-point Fourier

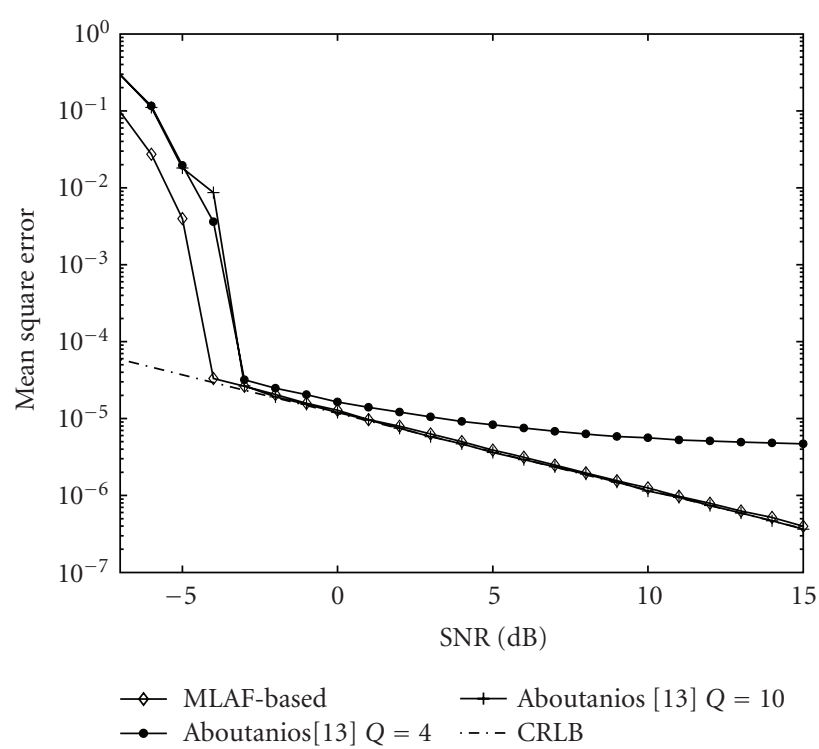

Figure 6: Comparison with periodogram based estimators. Signal frequency randomly distributes between $[0,0.5 \pi] . N=80$. For the MLAF-based estimator, $q=4$ and $\tau=1$.

Transform. If $M$ is a power of 2 , the DFT can be implemented using FFT, which requires $(M / 2) \log _{2} M$ complex operations. And in practice FFT can be implemented with fast hardware. The computations of the fine estimation stage for the proposed estimator are carried out inside the narrowband and its computational load is small. Furthermore, it is a closed-formed estimator.

\section{Conclusions}

In this paper, we present a new estimator based on main lobe autocorrelation functions. Performance analysis showed that the upper bound of the mean square error of the proposed estimator is 1.3 times of the CRLB for low to medium SNR. Furthermore, the proposed estimator has a full frequency range of $[-\pi, \pi)$. Simulations and analysis showed that the proposed estimator outperforms other existing autocorrelation based estimators.

\section{Appendix}

\section{A. Error Caused by Noise}

Since $M$ is large, we have $\sin \left(\left(k \omega_{0}-\omega_{c}\right) \tau\right) \cong\left(k \omega_{0}-\omega_{c}\right) \tau$. Hence, with (14) we have the error caused by noise:

$$
\begin{aligned}
& \frac{1}{\tau^{2}} E\left[P_{\tau, i}^{2}\right] \cong E\left[\left(\frac{\mathfrak{c}}{\sum_{k=0}^{M-1}\left(a_{k}^{2}+b_{k}^{2}\right)}\right)^{2}\right], \\
& \tau^{2} E\left[P_{\tau, i}^{2}\right]<2 E\left[\left(\frac{\mathfrak{d}}{\sum_{k=0}^{M-1}\left(a_{k}^{2}+b_{k}^{2}\right)}\right)^{2}\right],
\end{aligned}
$$


where $\mathfrak{c}$ denotes $\sum_{k=-\Delta}^{\Delta}\left(2 a_{k+k_{0}} \delta_{a_{k+k_{0}}}+2 b_{k+k_{0}} \delta_{b_{k+k_{0}}}+\delta_{a_{k+k_{0}}}^{2}+\right.$ $\left.\delta_{b_{k+k_{0}}}^{2}\right)\left(k \omega_{0}-\omega_{c}\right)$ and $\mathfrak{d}$ denotes $\sum_{k=-\Delta}^{\Delta}\left(2 a_{k+k_{0}} \delta_{a_{k+k_{0}}}+\right.$ $\left.2 b_{k+k_{0}} \delta_{b_{k+k_{0}}}\right)\left(k \omega_{0}-\omega_{c}\right)$. Obviously, (A.2) can be unwrapped as

$$
\tau^{2} E\left[P_{\tau, i}^{2}\right]<2 E\left(\delta_{1}+\delta_{2}\right)
$$

where

$$
\begin{aligned}
& \delta_{1}=8\left(\frac{\sum_{i=-\Delta}^{\Delta} \mathfrak{s}}{\left(\sum_{k=0}^{M-1}\left(a_{k}^{2}+b_{k}^{2}\right)\right)^{2}}\right), \\
& \delta_{2}=\frac{4 \sum_{i=-\Delta}^{\Delta} \mathfrak{e}}{\left(\sum_{k=0}^{M-1}\left(a_{k}^{2}+b_{k}^{2}\right)\right)^{2}},
\end{aligned}
$$

where $\mathfrak{s}$ denotes $\sum_{j=-\Delta}^{\Delta} a_{i^{\prime}}\left(i \omega_{0}-\omega_{c}\right) b_{j^{\prime}}\left(j \omega_{0}-\omega_{c}\right) E\left[\delta_{a_{i^{\prime}}} \delta_{b_{j^{\prime}}}\right]$ and $\mathfrak{e}$ denotes $\sum_{j=-\Delta}^{\Delta}\left(i \omega_{0}-\omega_{c}\right)\left(j \omega_{0}-\omega_{c}\right)\left(a_{i^{\prime}} a_{j^{\prime}}+\right.$ $\left.b_{i^{\prime}} b_{j^{\prime}}\right) E\left[\delta_{a_{i^{\prime}}} \delta_{a_{j^{\prime}}}\right]$ and $i^{\prime}=k_{0}+i, j^{\prime}=k_{0}+j . E\left[\delta_{a_{i^{\prime}}} \delta_{b_{j^{\prime}}}\right]$. $E\left[\delta_{a_{i^{\prime}}} \delta_{a_{j^{\prime}}}\right]$ and $E\left[\delta_{b_{i^{\prime}}} \delta_{b_{j^{\prime}}}\right]$ are expectations of correlations between spectral noises. They can be obtained with the definition of Fourier transform:

$$
\begin{aligned}
& E\left[\delta_{a_{i^{\prime}}} \delta_{b_{j^{\prime}}}\right]=E\left[z_{r n}^{2}\right] \Sigma_{s, \lambda}, \\
& E\left[\delta_{a_{i^{\prime}}} \delta_{a_{j^{\prime}}}\right]=E\left[\delta_{b_{i^{\prime}}} \delta_{b_{j^{\prime}}}\right]=E\left[z_{r n}^{2}\right] \Sigma_{c, \lambda}, \\
& \Sigma_{s, \lambda}= \begin{cases}\frac{q N \sin (\pi \lambda / q) \sin (\pi \lambda(N-1) / q N)}{\pi \lambda}, & \lambda \neq 0, \\
0, & \lambda=0,\end{cases} \\
& \Sigma_{c, \lambda}= \begin{cases}\frac{q N \sin (\pi \lambda / q) \cos (\pi \lambda(N-1) / q N)}{\pi \lambda}, & \lambda \neq 0, \\
N, & \lambda=0,\end{cases}
\end{aligned}
$$

where $\lambda=i-j . z_{r n}$ is the imaginary component of white noise $z(n)$ and $E\left[z_{r n}^{2}\right]=\sigma_{n}^{2} / 2$. To simplify computations, we perform samples' Fourier transform as follows:

$$
\begin{aligned}
X_{k} & =\sum_{n=-N / 2}^{N / 2-1} A e^{j \omega n} e^{-j k \omega_{0} n} \\
& =A \cdot \frac{\sin \left(\left(\omega-k \omega_{0}\right) N / 2\right)}{\sin \left(\left(\omega-k \omega_{0}\right) / 2\right)} e^{j\left(k \omega_{0}-\omega\right) / 2} .
\end{aligned}
$$

Substituting (A.6)-(A.10) into (A.2), and with some calculations we have

$$
\tau^{2} E\left[P_{\tau, i}^{2}\right]<2\left(\frac{8 q+4}{\gamma N^{3} q^{2}}+\frac{8}{\gamma}\left(\frac{(q-1 / 2) \Upsilon_{q}}{N^{3} q}\right)\right),
$$

where

$$
\Upsilon_{q}=\frac{\sum_{\lambda=1}^{2 q} \sin (2 \pi \lambda / q) \cos (\pi \lambda / q)}{\pi \lambda}
$$

\section{B. Error Caused by Main Lobe Autocorrelation Estimation}

According to (10) we can obtain

$$
\frac{\sum_{k \in[0, M-1]}\left(a_{k}^{2}+b_{k}^{2}\right) \sin \left(\left(\left(k-k_{0}\right) \omega_{0}-\omega_{c}\right) \tau\right)}{\sum_{k \in[0, M-1]}\left(a_{k}^{2}+b_{k}^{2}\right)}=0 .
$$

Thus with (B.1) and (15) we have

$$
\begin{aligned}
\frac{1}{\tau^{2}} Q_{\tau, i}^{2} & \cong-\left(\frac{\sum_{k=0, k \notin\left[k_{0}-\Delta, k_{0}+\Delta\right]}^{M-1}\left(a_{k}^{2}+b_{k}^{2}\right) \mathfrak{f}}{\sum_{k=0}^{M-1}\left(a_{k}^{2}+b_{k}^{2}\right)}\right)^{2}, \\
& \cong \frac{1}{\tau^{2}}\left[\left(\frac{\sum_{k \in[-\Delta, \Delta]}\left(a_{k+k_{0}}^{2}+b_{k+k_{0}}^{2}\right) \mathfrak{g}}{\sum_{k=0}^{M-1}\left(a_{k}^{2}+b_{k}^{2}\right)}\right)^{2}\right],
\end{aligned}
$$

where $\mathfrak{f}$ denotes $\sin \left(\left(\left(k-k_{0}\right) \omega_{0}-\omega_{\mathrm{c}}\right) \tau\right)$ and $\mathfrak{g}$ denotes $\sin \left(\left(k \omega_{0}-\omega_{c}\right) \tau\right)$. Then with (A.10), (B.2), the mean square error due to main lobe autocorrelation estimation yields

$$
\tau^{2} Q_{\tau, i}^{2} \approx\left(\frac{4}{N M} E\left[\sum_{k=-\Delta}^{\Delta} \frac{\sin ^{2}\left(\left(\omega_{c}-k \omega_{0}\right) N / 2\right)}{\left(\omega_{c}-k \omega_{0}\right)}\right]\right)^{2} .
$$

Comparing (7), (8) and (5), we note that $\omega_{c}$ can be expressed by $\omega_{c}=\alpha \omega_{0}$ where $\alpha=\omega_{c} M / 2 \pi$ uniformly distributes between $[-0.5,0.5]$. Thus, with (B.3) and $M=N q$, we have

$$
\tau^{2} Q_{\tau, i}^{2} \approx \frac{4}{q^{2} N^{2}}\left(R_{\sin c}(q, \alpha)\right)^{2}
$$

where

$$
R_{\sin c}(q, \alpha)=E\left[\sum_{k=-q}^{q} \frac{q \sin ^{2}(\pi(k-\alpha) / q)}{(k-\alpha)}\right] .
$$

\section{Acknowledgments}

The authors would like to show their sincerely appreciation to the anonymous reviewers for their very contributive comments in making the paper more appealing. This work was supported by the National Natural Science Foundation of China (no. 60625101, no. 60901070) and the Natural Science Foundation of Guangdong Province, China (no. 8151064101000066, no. 07006488).

\section{References}

[1] D. C. Rife and R. R. Boorstyn, "Single tone parameter estimation from discrete-time observations," IEEE Transactions on Information Theory, vol. 20, no. 5, pp. 591-598, 1974.

[2] Z. G. Zhang, S. C. Chan, and K. M. Tsui, "A recursive frequency estimator using linear prediction and a Kalmanfilter-based iterative algorithm," IEEE Transactions on Circuits and Systems II, vol. 55, no. 6, pp. 576-580, 2008.

[3] L. B. Jackson and D. W. Tufts, "Frequency estimation by linear prediction," in Proceedings of the IEEE International Conference on Acoustics, Speech and Signal Processing (ICASSP '78), pp. 352-356, Tulsa, Okla, USA, 1978. 
[4] G. Lank, I. Reed, and G. Pollon, "A semicoherent detection and Doppler estimation statistic," IEEE Transactions on Aerospace and Electronic Systems, vol. 9, no. 2, pp. 151-165, 1973.

[5] M. Fitz, "Further results in the fast estimation of a single frequency," IEEE Transactions on Communications, vol. 42, no. 234, part 2, pp. 862-864, 1994.

[6] M. Luise and R. Reggiannini, "Carrier frequency recovery in all-digital modems for burst-mode transmissions," IEEE Transactions on Communications, vol. 43, no. 2, pp. 1169$1178,1995$.

[7] Y. C. Xiao, P. Wei, X. C. Xiao, and H. M. Tai, "Fast and accurate single frequency estimator," Electronics Letters, vol. 40, no. 14, pp. 910-911, 2004.

[8] B. Volcker and P. Handel, "Frequency estimation from proper sets of correlations," IEEE Transactions on Signal Processing, vol. 50, no. 4, pp. 791-802, 2000.

[9] T. Brown and M. M. Wang, "An iterative algorithm for singlefrequency estimation," IEEE Transactions on Signal Processing, vol. 50, no. 11, pp. 2671-2682, 2002.

[10] U. Mengali and M. Morelli, "Data-aided frequency estimation for burst digital transmission," IEEE Transactions on Communications, vol. 45, no. 1, pp. 23-25, 1997.

[11] A. Awoseyila, C. Kasparis, and B. Evans, "Improved single frequency estimation with wide acquisition range," Electronics Letters, vol. 44, no. 3, pp. 245-247, 2008.

[12] B. G. Quinn, "Estimating frequency by interpolation using Fourier coefficient," IEEE Transactions on Signal Processing, vol. 42, no. 5, pp. 1264-1268, 1994.

[13] E. Aboutanios, "A modified dichotomous search frequency estimator," IEEE Signal Processing Letters, vol. 11, no. 2, pp. 186-188, 2004.

[14] E. Aboutanios and B. Mulgrew, "Iterative frequency estimation by interpolation on Fourier coefficients," IEEE Transactions on Signal Processing, vol. 53, no. 4, pp. 1237-1242, 2005.

[15] G. Wei, C. Yang, and F. J. Chen, "Closed-form frequency estimator based on narrow-band approximation," submitted.

[16] P. Handel, A. Eriksson, and T. Wigren, "Performance analysis of a correlation based single tone frequency estimator," Signal Processing, vol. 44, no. 2, pp. 223-231, 1995. 